\title{
Safe navigation strategies for a biped robot walking in a crowd
}

\author{
Néstor Bohórquez, Alexander Sherikov, Dimitar Dimitrov, Pierre-Brice Wieber \\ INRIA Rhône-Alpes, \\ 38334 Montbonnot Cedex, France \\ E-mail: \{nestor-alonso.bohorquez-dorante, alexander.sherikov, pierre-brice.wieber\}@inria.fr, mail@drdv.net
}

\begin{abstract}
Safety needs to be guaranteed before we can introduce robots into our working environments. For a biped robot to navigate safely in a crowd it must maintain balance and avoid collisions. In highly dynamic and unpredictable environments like crowds, collision avoidance is usually interpreted as passive safety, i.e. that the robot can stop before any collision occurs. We show that both balance preservation and passive safety can be analyzed, from the point of view of viability theory, as the ability of the robot to stop safely at some point in the future. This allows us to address both problems with a single model predictive controller with appropriate terminal constraints. We demonstrate that this controller predicts failures (falls and collisions) as early as the duration of the preview horizon. Finally, we propose a new strategy for safe navigation that relaxes the passive safety conditions to allow the robot to avoid a greater number of collisions.
\end{abstract}

\section{INTRODUCTION}

The objective in this paper is to make biped robots walk safely in a human crowd. This presents two particular problems: maintaining balance and avoiding collisions. It has already been observed that both problems can be addressed with the concept of viability [1]. In viability theory [2], the objective is to ensure that the system avoids undesirable states in the future. In our case, the undesirable states are those where the robot has fallen or collided with somebody. The difficulty resides in achieving these objectives with limited knowledge of the environment and of the future, specially of the future motion of people around the robot.

If we can make sure that the robot can stop in a few steps in a stable position (i.e. that it is capturable [3], [4]), then it can keep its balance indefinitely. This can be accomplished with limited knowledge of the environment and it has proven to be compatible with the realization of highly dynamic motions [5]. The case of collision avoidance is more challenging, as we cannot guarantee it indefinitely, specially in a crowd with fast moving persons. We can ensure, however, passive safety [6], [7], i.e. that the robot is able to stop before any collision occurs. This corresponds to the emergency stop procedures required for industrial and personal care robots [8], [9]. We can observe that the capacity to stop is integral to both concepts and, thanks to this, passive safety can be combined effortlessly with capturability. In our approach, we incorporate these two concepts in a single Model Predictive Control (MPC) scheme [10].

There are situations where it is not possible for the robot to avoid a fall or a collision. It is fundamental to predict these situations as early as possible for the safety of the people around, of the robot and of the workspace [11], [12]. By previewing the short-term future, our proposed controller predicts failures before they occur. We can make the anticipation time of such events arbitrarily early (by previewing further into the future) provided that the perception capabilities of the robot satisfy some minimal conditions related to the speed at which the environment evolves.

Lastly, we propose a new strategy for safe navigation in dynamic environments that builds upon the observation that the stop requirement in passive safety can limit the mobility of the robot (specially in close proximity of people). This behavior is accentuated as uncertainty in the perception of the environment increases. We demonstrate that it produces fewer collisions than passive safety and, when they happen, they are less severe.

We proceed as follows: we briefly describe the basis for our numerical approach to safe navigation in Section II. Then we introduce the elements of the problem of walking in a crowd in Section III. We formulate two MPC controllers for passive safety and two for relaxed passive safety in Section IV. We evaluate the performance of all strategies through numerical simulations whose results we present in Section V.

\section{LEXICOGRAPHIC OPTIMIZATION FOR SAFE NAVIGATION}

To achieve passive safety, a biped robot has to maintain balance and be able to stop before any collision happens. Additionally, we also want the robot to follow a reference velocity. But these objectives might conflict and, in such cases, at least one of them has to be relaxed [13]. To do this, we express all objectives as linear inequalities on the state $x$ of the robot

$$
\underline{b} \leq A x \leq \bar{b},
$$

where equalities can be expressed with $\underline{b}=\bar{b}$. Relaxation can be done, then, by introducing a violation $v$ as in

$$
\underline{b} \leq A x-v \leq \bar{b},
$$

and minimizing $\|v\|^{2}$. Note that the objective is satisfied if $\|v\|=0$.

Deciding which objectives can be relaxed is problemdependent. In our case, avoiding collisions and maintaining balance are more important objectives than following a 
reference velocity. We can assign a priority to each objective and minimize their violations accordingly, that is, as a lexicographic least squares problem [14], [15], [16]. In order to do this, we define $P$ levels of priorities, each one described by a triplet $\left(A_{i}, \underline{b_{i}}, \overline{b_{i}}\right)$, and we look to

$$
\begin{aligned}
\text { lex } & \operatorname{minimize} \\
\text { subject to } & \left(\left\|v_{1}\right\|^{2}, \ldots,\left\|v_{P}\right\|^{2}\right) \\
& {\left[\begin{array}{c}
\frac{b_{1}}{\vdots} \\
b_{P}
\end{array}\right] \leq\left[\begin{array}{c}
A_{1} \\
\vdots \\
A_{P}
\end{array}\right] x-\left[\begin{array}{c}
v_{1} \\
\vdots \\
v_{P}
\end{array}\right] \leq\left[\begin{array}{c}
\overline{b_{1}} \\
\vdots \\
\overline{b_{P}}
\end{array}\right] . }
\end{aligned}
$$

Lexicographic optimization is a key aspect of our approach to safe navigation, as will be explained in the following sections.

\section{MODELS OF THE ROBOT AND THE CROWD}

In this section, we first discuss the models that we consider for the anticipation of motions of the robot and the crowd. We then formalize the safety criteria for navigation (dynamic and kinematic feasibility, collision avoidance and stopping conditions) in the context of these models.

\section{A. Dynamic and kinematic feasibility}

A biped robot is a complex, nonlinear, underactuated, hybrid dynamical system [17]. However, we can generate standard walking motions (i.e. on a flat ground with a constant height of the Center of Mass (CoM)) using a linear relation between the horizontal motion of the CoM and the Center of Pressure $(\mathrm{CoP})$ on the ground:

$$
c-\frac{h}{g} \ddot{c}=p .
$$

In this equation, $c \in \mathbb{R}^{2}$ and $p \in \mathbb{R}^{2}$ are the horizontal positions of the CoM and CoP respectively, $h$ is the height of the CoM and $g$ is the norm of the gravity vector. We consider only this simple situation in this paper, although walking motion can be generated on uneven ground with varying height of the CoM as well using similar linear approaches [18].

Due to the unilaterality of the contact forces with the ground, the CoP is always constrained to stay inside the support polygon [19]:

$$
p \in \mathcal{P}(s)
$$

where $\mathcal{P}(s)$ is a convex set that is defined relative to the center of the foot on the ground $s \in \mathbb{R}^{2}$. In combination with (4), this limits the acceleration that the CoM can achieve.

We enforce a kinematic constraint related to the maximal leg length of the robot:

$$
\|c-s\| \leq L
$$

Moreover, most biped robots cannot cross their legs when walking. Consequently, we restrict the position of the $(j+$ $1)^{t h}$ footstep with respect to the $j^{t h}$ to a region where this cannot happen:

$$
s_{j+1} \in \mathcal{S}\left(s_{j}\right)
$$

where $\mathcal{S}\left(s_{j}\right)$ is a convex set defined relative to $s_{j}$.

We state that the motion of a biped robot is dynamically and kinematically feasible if the following expression holds:

$$
\text { Feasibility } \equiv(4) \wedge(5) \wedge(6) \wedge(7)
$$

We linearize the constraints (5), (6) and (7) in our implementation by making standard assumptions on the kinematics of the robot [20], [21].

\section{B. Model of the crowd and collision avoidance}

Let us consider a crowd of $M$ persons. In order to avoid collisions, we enforce a minimal separation distance between the robot and each person:

$$
\text { Collision avoidance } \equiv\left\|c-m_{k}\right\| \geq d
$$

for all $k \in\{1, \ldots, M\}$. In this equation, $m_{k} \in \mathbb{R}^{2}$ is the position of the $k^{t h}$ person and $d$ is the minimal separation distance.

Passive safety requires the robot to avoid collisions in the present and in the future, until it is able to stop. We need, therefore, a model to anticipate the motion of people. We make the following assumptions: people walk at constant velocity (at least during the short period of time before the robot is able to stop) so they do not try to avoid the robot; and collisions among people are disregarded.

We also take into account that the robot perception of the crowd has some degree of uncertainty. Consequently, the estimated position $\hat{m}_{k}$ and velocity $\hat{\dot{m}}_{k}$ of each person have associated errors:

$$
\tilde{m}_{k}=m_{k}-\hat{m}_{k}, \quad \tilde{\dot{m}}_{k}=\dot{m}_{k}-\hat{\dot{m}}_{k},
$$

with respect to the real position $m_{k}$ and velocity $\dot{m}_{k}$. We respond to this uncertainty by adapting the minimal separation distance in a conservative way:

$$
d(t)=d_{0}+\left\|\tilde{m}_{k}\right\|+\left\|\tilde{\dot{m}}_{k}\right\| t .
$$

Here, given the magnitude of the uncertainty in position $\left\|\tilde{m}_{k}\right\|$, we consider that the real position $m_{k}$ lies in a circle of radius $\left\|\tilde{m}_{k}\right\|$ centered at the estimated position $\hat{m}_{k}$. Furthermore, given the magnitude of the uncertainty in velocity $\left\|\tilde{\dot{m}}_{k}\right\|$, the radius of this circle increases at a rate $\left\|\tilde{\dot{m}}_{k}\right\| t$ as it moves with the estimated velocity $\hat{\dot{m}}_{k}$. Figure 1 shows two examples of how uncertainty shapes our model of the future.

In our implementation, we linearize the constraint on collision avoidance $(C)$ as follows:

$$
\hat{u}_{k}^{T}\left(c-m_{k}\right) \geq d
$$

where

$$
\hat{u}_{k}=\frac{\left(c-m_{k}\right)}{\left\|c-m_{k}\right\|} \in \mathbb{R}^{2}
$$

is a unit normal vector that points from the center of the $k^{t h}$ person to the CoM of the robot. An advantage of this approach is that, by being conservative, this linear approximation is always safe with respect to the nonlinear problem, as can be observed in Figure 2. 

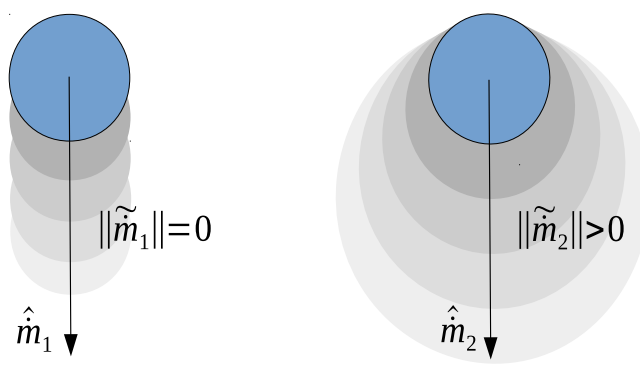

Fig. 1: Two different predictions of the motion of a person: without uncertainty (left) and with uncertainty in velocity (right). The gray areas are the predicted future positions of the person. When there is uncertainty in velocity, the set of possible positions the person could be in the future increase with time, following (9).

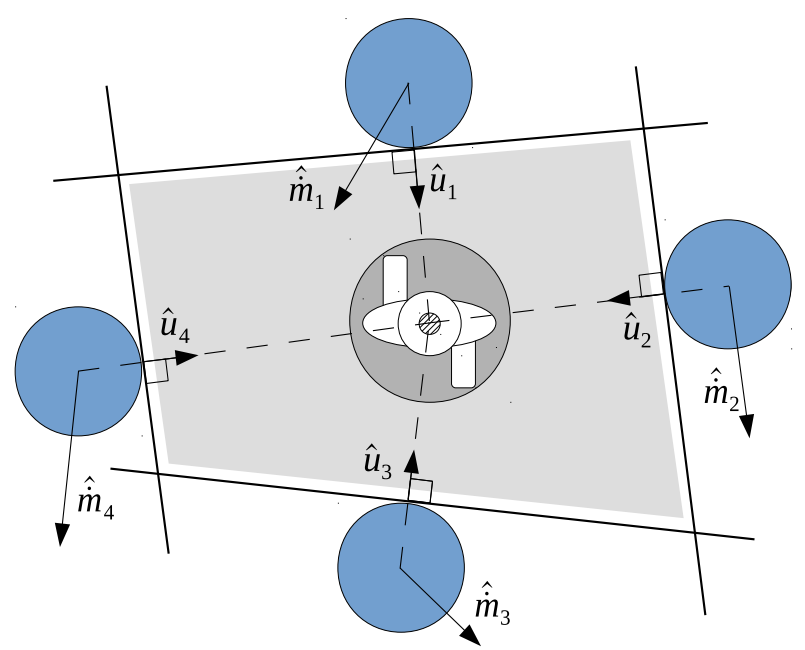

Fig. 2: Collision avoidance. The vectors $\hat{u}_{k}$ define lines that the robot cannot cross. These lines create a convex hull (the light gray area) where the robot is allowed to move.

\section{Stop condition}

We can determine trivially from (4) and (5) that the robot has stopped when $\dot{c}=\ddot{c}=0$ and $c=p \in \mathcal{P}(s)$. It is also possible to identify situations where the robot has not stopped yet but can stop without having to make any further step, i.e. where it is 0 -step capturable [4], by introducing the following constraint on the Capture Point:

$$
\text { Stop } \equiv c+\sqrt{\frac{h}{g}} \dot{c} \in \mathcal{P}(s)
$$

This will be our stop condition.

\section{Walking objectives}

In addition to these constraints on dynamic and kinematic feasibility, collision avoidance and stopping condition, we would also like the CoM to follow, as much as possible, a

\section{Hierarchy 1: Passive safety}

1: Passive safety conditions:

- Feasibility: $F_{i}, \forall i \in \mathcal{I}$

- Collision avoidance: $C_{i}, \forall i \in \mathcal{I}$

- Stop at the end: $S_{N}$

2: Objectives: $O_{i}, \forall i \in \mathcal{I}$

reference walking velocity $\dot{c}_{r e f}$ [21]. Furthermore, we are interested in keeping the $\mathrm{CoP}$ as close as possible to the center of the foot on the ground $s$ to improve the robustness of the robot against perturbations [22]. We refer to these as:

$$
\text { Objectives } \equiv\left\{\begin{array}{l}
\dot{c}=\dot{c}_{\text {ref }} \\
p=s
\end{array}\right.
$$

\section{SAFE NAVIGATION STRATEGIES}

In this Section, we discuss three different ways we can integrate the constraints of the robot to formulate safe navigation controllers.

\section{A. Passive safety: triggering an emergency stop}

Given a horizon of $N$ sampling times into the future $\mathcal{I}=\{1, \ldots, N\}$, preserving passive safety means that the robot must satisfy the stop condition $(S)$ at the $N^{t h}$ sampling time, what we note here $S_{N}$. It must also satisfy the constraints on dynamic and kinematic feasibility $(F)$ and collision avoidance $(C)$ at all time until then, i.e. $F_{i}$ and $C_{i}$ for all $i \in \mathcal{I}$. Once these three conditions are met, the remaining degrees of freedom can be used to satisfy, as much as possible, the objectives $(O)$, i.e. $O_{i}$ for all $i \in \mathcal{I}$. We can formalize the hierarchy between the constraints and the objectives as the lexicographic least squares problem summarized in Hierarchy 1.

The solution to this lexicographic problem provides the robot with a trajectory to walk safely and stop after $N$ sampling periods. In order to generate a steady walking motion, we implement a receding horizon strategy: the lexicographic problem is re-evaluated at each sampling time for a horizon of $N$ sampling periods in the future, continuously postponing the moment when the robot would eventually stop.

If it is not possible to safely postpone the moment when the robot stops, it can simply follow the last safe plan and stop before any collision occurs. When this happens, we raise an alarm to indicate that the robot is initiating an emergency stop and that it is not safe to continue walking. This alarm can be used to warn the surrounding crowd of the risk of collision $N$ sampling periods in the future.

\section{B. Passive safety with deferrable emergency stop}

During the emergency stop, the robot will steadily approach the moment planned for the stop and the horizon will shrink accordingly. It might be beneficial, however, to continue re-evaluating the situation to check if, based on 


\section{Hierarchy 2: Relaxed passive safety 1}

1: Feasibility: $F_{i}, \forall i \in \mathcal{I}$

2: Collision avoidance: $C_{1}$

N: Collision avoidance: $C_{N-1}$

N+1: Collision avoidance: $C_{N}$, Stop: $S_{N}$

N+2: Objectives: $O_{i}, \forall i \in \mathcal{I}$

\section{Hierarchy 3: Relaxed passive safety 2}

1: Feasibility: $F_{1}$, Collision avoidance: $C_{1}$ :

N-1: Feasibility: $F_{N-1}$ Collision avoidance: $C_{N-1}$

N: Feasibility: $F_{N}$, Collision avoidance: $C_{N}$, Stop: $S_{N}$

N+1: Objectives: $O_{i}, \forall i \in \mathcal{I}$

new measurements of the surrounding crowd, it is possible to postpone the stop of the robot, as long as possible.

We can go through a simple loop, at each new sampling period, to find the largest $N$ (up to a nominal value) such that all feasibility, collision avoidance and stopping condition are satisfied.

\section{Relaxed passive safety}

As soon as the robot is unable to plan a new safe motion, passive safety requires that it initiates an emergency stop. But in case of uncertainty, our conservative approach (see (9) and Figure 1) might exaggerate the risk of collision, leading to inappropriate stops. Another strategy could be to satisfy all passive safety constraints for as long as possible and, when not possible, relax them in order to produce a solution that is as close as possible to passive safety. In this way, the robot tries to minimize the risk of collision instead of simply initiating an emergency stop.

Building on our lexicographic optimization approach, we propose a relaxation of constraints along the horizon: we try to satisfy first the constraints in the near present and then, if possible, those in the far future. In particular, we can try to satisfy $C_{i}$ and, if possible, $C_{i+1}$ for all $i \in\{1, \ldots, N\}$. Correspondingly, the stop requirement $S_{N}$ and the objectives would belong now to the lowest levels. Hierarchy 2 shows how we order these priorities in a single formulation. In this case, all dynamic and kinematic feasibility constraints appear at the highest priority level, but the same relaxation scheme could be applied as well, i.e. $F_{i} \succ F_{i+1}$ for all $i \in\{1, \ldots, N\}$. Hierarchy 3 shows how we order the temporal relaxation of both feasibility and collision avoidance.

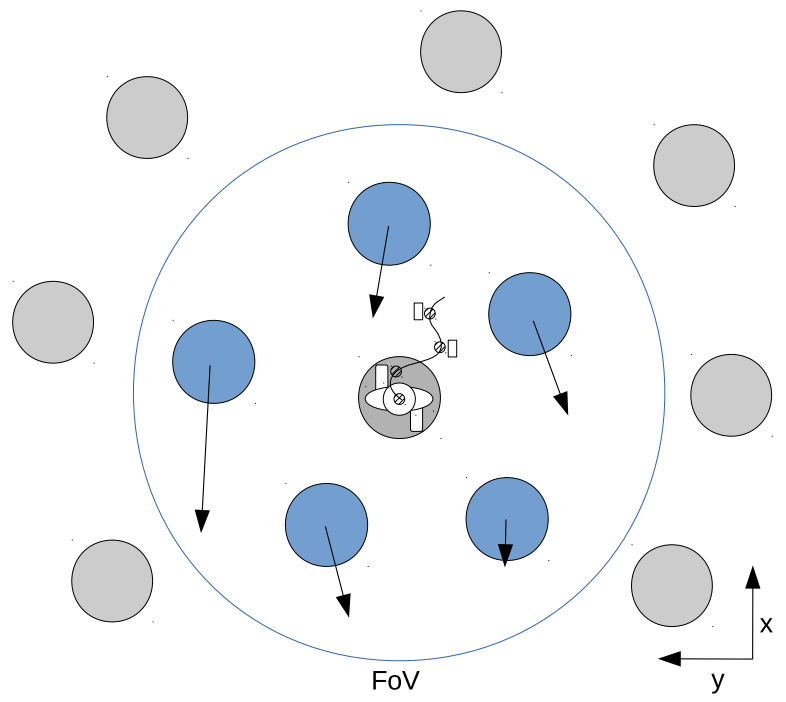

Fig. 3: Walking against a crowd.

A similar approach can be used to generate time-optimal trajectories for manipulator robots [23].

\section{EVALUATION OF THE SAFE NAVIGATION STRATEGIES}

We evaluate numerically the performance of the proposed safe navigation strategies by making the robot traverse a moving crowd (as shown in Figure 3) under different environmental conditions and parameters of the controller. Table I shows the parameters for the kinematics of our simulated robot, which is based on the HRP-2 [24]. Tables II and III show the parameters of the two scenarios of our numerical evaluations.

For each combination of size and speed along $x\left(M, \dot{m}_{k}^{x}\right)$ we generate and store 100 different crowds that only differ in the initial positions $\left\{m_{k}\right\}$ and speeds along $y\left\{\dot{m}_{k}^{y}\right\}$ of the participants. The initial positions vary uniformly over an area of $10 \times 8\left[\mathrm{~m}^{2}\right]$ while speeds along $y$ follow a normal distribution $\mathcal{N}(0,0.2)$.

During simulations, we test each configuration of the controller $\left(H, R,\left\|\tilde{m}_{k}\right\|,\left\|\tilde{\dot{m}}_{k}\right\|\right)$ against each of the 100 crowds generated for each combination $\left(M, \dot{m}_{k}^{x}\right)$. Simulations last $20[s]$ or until a failure occurs, i.e. when the state of the robot is infeasible or when it collides with a person.

We use the notion of a Field of View (FoV): the maximal distance around the robot at which it is capable of perceiving persons/objects. We do not consider occlusions in perception, making the robot aware of the current position and velocity of everybody within the FoV.

\section{A. No uncertainty in the motion of the crowd}

Figure 4 synthesizes the results obtained from all simulations with no uncertainty in the motion of the crowd. Plots on the left show the failure rate as a function of the radius of the FoV and for different walking speeds of the crowd. Plots on the right report the corresponding median anticipation time to failure, which is the elapsed time between the moment the earliest alarm was triggered and the moment the failure 
TABLE I: Parameters of biped robot

\begin{tabular}{cccc}
\hline Parameter & Symbol & Value & Unit \\
\hline Height of CoM (4) & $h$ & 0.80 & $\mathrm{~m}$ \\
Feet dimensions (5) & $Z$ & $(0.24,0.14)$ & $(\mathrm{m}, \mathrm{m})$ \\
Leg stride (6) & $L$ & 0.30 & $\mathrm{~m}$ \\
Feet separation (7) & $D$ & 0.20 & $\mathrm{~m}$ \\
Safety distance (9) & $d_{0}$ & 1 & $\mathrm{~m}$ \\
Reference speed (O) & $\dot{c}_{r e f}^{x}$ & 0.5 & $\mathrm{~ms}^{-1}$ \\
Step duration & $\dot{c}_{r e f}^{y}$ & 0 & $\mathrm{~ms}^{-1}$ \\
Sampling time & - & 0.8 & $\mathrm{~s}$ \\
\hline
\end{tabular}

TABLE II: Parameters of simulations with no uncertainty

\begin{tabular}{cccc}
\hline Parameter & Symbol & Value & Unit \\
\hline Horizon duration & $H$ & $\{1.0,1.8,2.6\}$ & $\mathrm{s}$ \\
Radius of FoV & $R$ & $\{0.5,1,2,3,4\}$ & $\mathrm{m}$ \\
Size of crowd & $M$ & $\{8,16,32\}$ & - \\
Speed of the crowd & $\dot{m}_{k}^{x}$ & $\{0.25,0.5,1,1.5,2\}$ & $\mathrm{ms}^{-1}$ \\
& $\dot{m}_{k}^{y}$ & {$[-0.2,0.2]$} & $\mathrm{ms}^{-1}$ \\
Uncertainty & $\left\|\tilde{m}_{k}\right\|$ & 0 & $\mathrm{~m}$ \\
& $\left\|\tilde{\dot{m}}_{k}\right\|$ & 0 & $\mathrm{~ms}^{-1}$ \\
\hline
\end{tabular}

happened. It is worth mentioning that, in these simulations with no uncertainty, all failures are anticipated as early as permitted by the limits of the FoV and the prediction horizon, and no false alarms are raised.

We can see two different regimes represented with either dashed lines or solid lines. The threshold is attained when the radius $R$ of the FoV reaches

$$
R_{\text {min }}=H\left(\dot{m}_{k}^{x}+\dot{c}_{r e f}^{x}\right),
$$

which is the minimum value for detecting all people that might collide with the robot over a horizon of duration $H$, with walking speeds $\dot{m}_{k}^{x}$ for the people and $\dot{c}_{r e f}^{x}$ for the robot.

If the FoV is smaller than this minimum value, the anticipation time for collisions grows linearly with the radius of the FoV and the failure rate decreases accordingly. It does not, however, decrease with longer prediction horizons: it appears that longer prediction horizons can degrade collision avoidance if the radius of the FoV is not large enough, as the robot is making decisions without proper knowledge of the surrounding crowd.

If the FoV is larger than this minimum value, the anticipation time for collisions reaches exactly the duration $H$ of the

TABLE III: Parameters of simulations with uncertainty

\begin{tabular}{cccc}
\hline Parameter & Symbol & Value & Unit \\
\hline Horizon duration & $H$ & 1.8 & $\mathrm{~s}$ \\
Radius of FoV & $R$ & 4 & $\mathrm{~m}$ \\
Size of crowd & $M$ & 16 & - \\
Speed of the crowd & $\dot{m}_{k}^{x}$ & 0.5 & $\mathrm{~ms}^{-1}$ \\
& $\dot{m}_{k}^{y}$ & {$[-0.2,0.2]$} & $\mathrm{ms}^{-1}$ \\
Uncertainty & $\left\|\tilde{m}_{k}\right\|$ & $\{0,0.15,0.30\}$ & $\mathrm{m}$ \\
& $\left\|\tilde{\dot{m}}_{k}\right\|$ & $\{0,0.05,0.10\}$ & $\mathrm{ms}^{-1}$ \\
\hline
\end{tabular}

prediction horizon, and the failure rate reaches a minimum, which does not vary further with the radius of the FoV.

When the robot walks in an inattentive crowd that moves at a standard human walking speed (between $1\left[\mathrm{~ms}^{-1}\right]$ and $\left.1.5\left[\mathrm{~ms}^{-1}\right][25]\right)$, the probability of collisions is always higher than $50 \%$. The robot does not have enough kinematic and dynamic capabilities to avoid collisions with people walking that fast. We can, however, anticipate these collisions as early as needed, with appropriate FoV and prediction horizon following the simple suggestions of (12), and initiate an emergency stop.

\section{B. Uncertainty in the motion of the crowd}

Tables IV and V show, for passive safety and relaxed passive safety strategies respectively, the number of failures that take place when there is uncertainty in the perception of people. In each table, the first two columns are the values of uncertainty. The next two columns present the median velocities of both the robot and the person at the time of collision along the direction of the contact. Cases with no collision are indicated with a dash "-". The last column shows the proportion of simulations that ended with a failure.

The failure rate of the emergency stop strategy varies little with the uncertainty in position but increases with the uncertainty in velocity. Uncertainty causes this strategy to make unnecessary and undesirable stops that lead to collisions. In the deferrable emergency stop strategy, the failure rate seems unaffected by the uncertainty in position as well but decreases with the uncertainty in velocity.

It is important to note that, in our model of the crowd, the motion of people does not become more erratic with increased uncertainty. What increases is the size of the areas where people might be in the future. With only uncertainty in position, these areas have equal size throughout the horizon. People appear to be larger and there is less space to evade them. With only uncertainty in velocity, these areas increase in size along the preview horizon. People appear to be larger at the distance but reduce their size as the robot approaches them.

The velocity of the robot at the moment of collision is either zero or close to zero but negative, as expected. This suggests that the robot is trying to walk away from people before the collision happens.

The controller that implements Hierarchy 3 walks away from people as much as possible, even by following trajectories that lead to infeasible future states. This results in fewer collisions than any other strategy only because the robot is deciding to fall on the ground instead. On the other hand, with the controller that implements Hierarchy 2, the order of the priorities requires the motion of the robot to be dynamically and kinematically feasible during the whole prediction. The resulting number of collisions is higher but the total number of failures is lower.

The controller that implements Hierarchy 2 delays collisions as much as possible. The corresponding optimal control action is, in most cases, to make the robot walk away from people while in some other specific cases (e.g. when people 

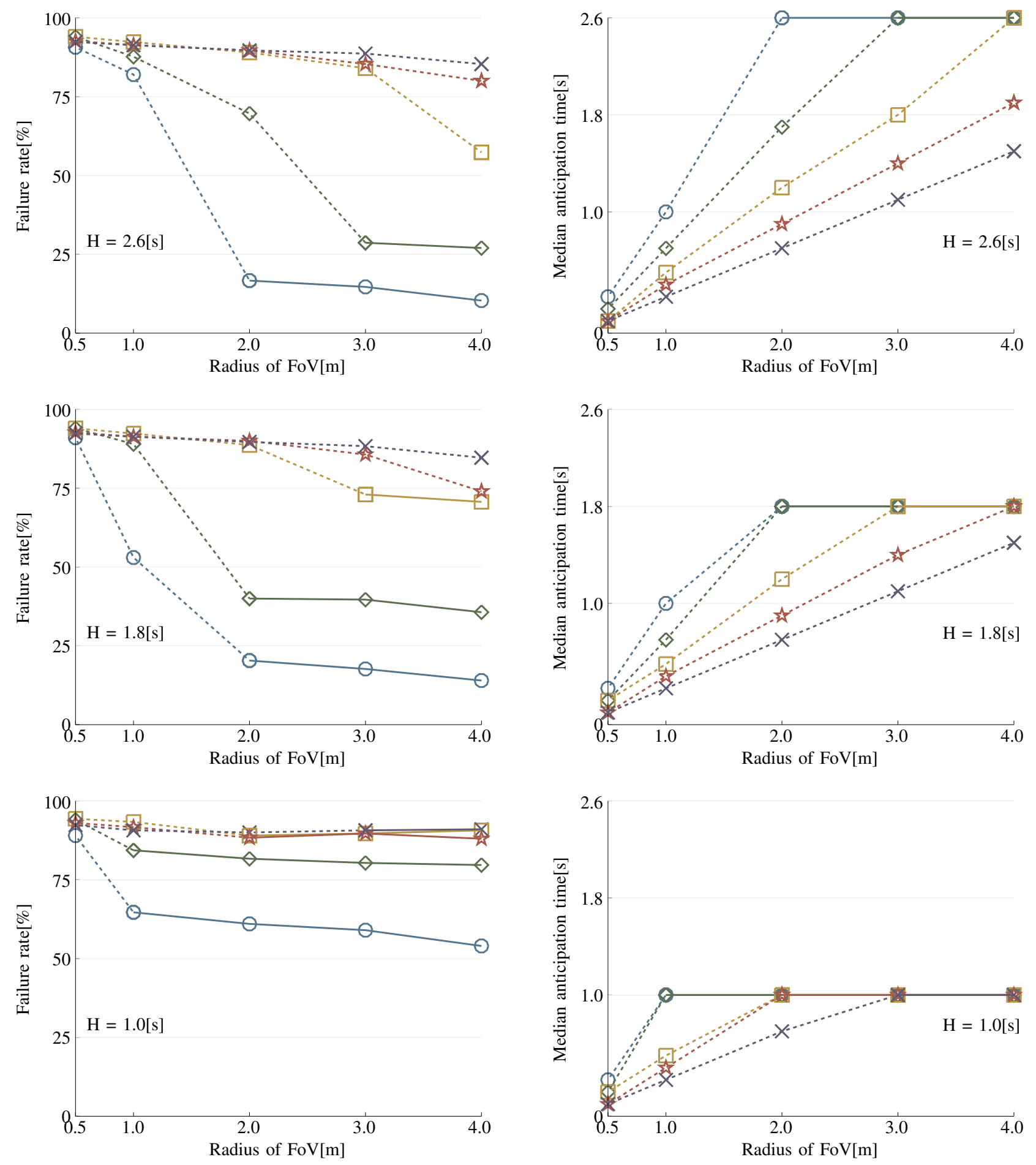

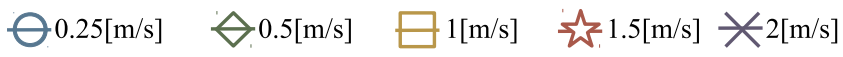

Fig. 4: Failure rate and median anticipation time for different speeds of the crowd and different durations of the horizon. 
TABLE IV: Collisions of passive safety strategies

\begin{tabular}{cccccc}
\hline \multicolumn{2}{c}{ Uncertainty } & & \multicolumn{2}{c}{ Collision vel $\left[\mathrm{ms}^{-1}\right]$} & $\begin{array}{c}\text { Failure } \\
\text { rate }[\%]\end{array}$ \\
\cline { 1 - 4 } Pos $[\mathrm{m}]$ & Vel $\left[\mathrm{ms}^{-1}\right]$ & Robot & Person & \\
\hline 0 & 0 & -0.04 & 0.48 & 27 \\
0 & 0.05 & 0.00 & 0.52 & 34 \\
0 & 0.10 & & 0.00 & 0.41 & 36 \\
0.15 & 0 & & -0.01 & 0.44 & 28 \\
0.15 & 0.05 & 0.00 & 0.40 & 38 \\
0.15 & 0.10 & 0.00 & 0.41 & 38 \\
0.30 & 0 & & 0.00 & 0.46 & 34 \\
0.30 & 0.05 & 0.00 & 0.50 & 40 \\
0.30 & 0.10 & 0.00 & 0.41 & 39 \\
\hline
\end{tabular}

\begin{tabular}{cccccc}
\hline \multicolumn{2}{c}{ Uncertainty } & & \multicolumn{2}{c}{ Collision vel $\left[\mathrm{ms}^{-1}\right]$} & $\begin{array}{c}\text { Failure } \\
\text { rate }[\%]\end{array}$ \\
${$\cline { 1 - 4 }$[\mathrm{m}]} }$ & Vel $\left[\mathrm{ms}^{-1}\right]$ & Robot & Person & \\
\hline 0 & 0 & & -0.05 & 0.36 & 32 \\
0 & 0.05 & & 0.00 & 0.55 & 15 \\
0 & 0.10 & 0.00 & 0.55 & 9 \\
0.15 & 0 & & -0.01 & 0.42 & 31 \\
0.15 & 0.05 & 0.00 & 0.52 & 15 \\
0.15 & 0.10 & 0.00 & 0.55 & 9 \\
0.30 & 0 & 0.00 & 0.45 & 35 \\
0.30 & 0.05 & 0.00 & 0.55 & 15 \\
0.30 & 0.10 & 0.00 & 0.53 & 9 \\
\hline
\end{tabular}

(a) Hierarchy 1: emergency stop

(b) Hierarchy 1: deferrable emergency stop

TABLE V: Collisions of relaxed passive safety strategies

\begin{tabular}{cccccc}
\hline \multicolumn{2}{c}{ Uncertainty } & & \multicolumn{2}{c}{ Collision vel $\left[\mathrm{ms}^{-1}\right]$} & \multirow{2}{*}{$\begin{array}{c}\text { Failure } \\
\text { rate }[\%]\end{array}$} \\
\cline { 1 - 2 } \cline { 5 - 6 } Pos $[\mathrm{m}]$ & Vel $\left[\mathrm{ms}^{-1}\right]$ & Robot & Person & \\
\hline 0 & 0 & -0.33 & 0.39 & 19 \\
0 & 0.05 & & -0.71 & 0.60 & 4 \\
0 & 0.10 & & -0.53 & 0.59 & 2 \\
0.15 & 0 & & -0.19 & 0.49 & 15 \\
0.15 & 0.05 & & -0.74 & 0.58 & 5 \\
0.15 & 0.10 & -0.64 & 0.58 & 2 \\
0.30 & 0 & & -0.14 & 0.56 & 15 \\
0.30 & 0.05 & & -0.48 & 0.58 & 5 \\
0.30 & 0.10 & -0.28 & 0.57 & 2 \\
\hline
\end{tabular}

(a) Hierarchy 2: relaxation of collision avoidance

\begin{tabular}{|c|c|c|c|c|}
\hline \multicolumn{2}{|c|}{ Uncertainty } & \multicolumn{2}{|c|}{ Collision vel $\left[\mathrm{ms}^{-1}\right]$} & \multirow{2}{*}{$\begin{array}{l}\text { Failure } \\
\text { rate[\%] }\end{array}$} \\
\hline $\operatorname{Pos}[\mathrm{m}]$ & $\operatorname{Vel}\left[\mathrm{ms}^{-1}\right]$ & Robot & Person & \\
\hline 0 & 0 & -0.60 & 0.39 & 21 \\
\hline 0 & 0.05 & - & - & 11 \\
\hline 0 & 0.10 & - & - & 13 \\
\hline 0.15 & 0 & - & - & 22 \\
\hline 0.15 & 0.05 & - & - & 10 \\
\hline 0.15 & 0.10 & - & - & 13 \\
\hline 0.30 & 0 & - & - & 27 \\
\hline 0.30 & 0.05 & - & - & 12 \\
\hline 0.30 & 0.10 & - & - & 13 \\
\hline
\end{tabular}

(b) Hierarchy 3: relaxation of collision avoidance and feasibility are coming towards the robot from all directions at the same speed) it is to make it remain still. In any case, the difference of velocities at the moment of collision is guaranteed to be no bigger than it would be if the robot was at rest. Collisions are, therefore, less severe.

The typical problem solved at every iteration by the relaxed passive safety controllers on the scenario shown in Table III contains: 530 constraints distributed along 20 priority levels and 40 decision variables. It is solved, on average, in $10[\mathrm{~ms}]$ on a computer with a $3[\mathrm{GHz}]$ Intel Core i7 processor.

\section{CONCLUSiONS}

We designed controllers for the safe navigation of biped robots in crowds merging viability concepts from balance and collision avoidance. We developed two passive safety controllers and a new strategy that addresses the limitations of passive safety. All schemes were evaluated in simulations of walking crowds where we: 1) made recommendations on the minimal perception requirements of the robot to navigate safely; 2) demonstrated that failures could be previewed as early as possible and with no false alarms in perfectly known environments; 3) attested that the relaxed passive safety strategy produces less collisions than passive safety ones and, when they happen, they are less severe.

Some aspects of this work can lead to further research: considering occlusions of people inside the FoV; reasoning about what happens after a collision takes place and how damages to people, the robot and the environment can be minimized.

\section{ACKNOWLEGMENTS}

This work has been funded by the PSPC Romeo 2 project and EU H2020 Comanoid Research and Innovation Action (RIA).

\section{REFERENCES}

[1] P.-B. Wieber, "Viability and Predictive Control for Safe Locomotion," in IEEE-RSJ International Conference on Intelligent Robots \& Systems, Nice, France, 2008.

[2] J.-P. Aubin, Viability theory. Birkhäuser, 1991.

[3] J. Pratt, J. Carff, S. Drakunov, and A. Goswami, "Capture point: A step toward humanoid push recovery," in Humanoid Robots, 2006 6th IEEE-RAS International Conference on, dec. 2006, pp. 200 -207.

[4] T. Koolen, T. de Boer, J. Rebula, A. Goswami, and J. Pratt, "Capturability-based analysis and control of legged locomotion, part 1: Theory and application to three simple gait models," The International Journal of Robotics Research, vol. 31, no. 9, pp. 1094-1113, 2012.

[5] A. Sherikov, D. Dimitrov, and P.-B. Wieber, "Whole body motion controller with long-term balance constraints," in Humanoid Robots (Humanoids), 2014 14th IEEE-RAS International Conference on, Nov 2014, pp. 444-450.

[6] K. Macek, D. A. Vasquez Govea, T. Fraichard, and R. Y. Siegwart, "Towards Safe Vehicle Navigation in Dynamic Urban Scenarios," Automatika, Nov. 2009.

[7] S. Bouraine, T. Fraichard, and H. Salhi, "Provably Safe Navigation for Mobile Robots with Limited Field-of-Views in Dynamic Environments," Autonomous Robots, vol. 32, no. 3, pp. 267-283, Apr. 2012.

[8] ISO, Robots and robotic devices - Safety requirements for industrial robots - Part 1: Robots, ser. ISO 10218-1:2011. ISO, Geneva, Switzerland, 2011.

[9] — Robots and robotic devices - Safety requirements for personal care robots, ser. ISO 13482:2014. ISO, Geneva, Switzerland, 2014. 
[10] D. Mayne, J. Rawlings, C. Rao, and P. Scokaert, "Constrained model predictive control: Stability and optimality," Automatica, vol. 36, no. 6, pp. $789-814,2000$.

[11] S. Kalyanakrishnan and A. Goswami, "Learning to predict humanoid fall," International Journal of Humanoid Robotics, vol. 08, no. 02, pp. 245-273, 2011.

[12] O. Hohn and W. Gerth, "Probabilistic balance monitoring for bipedal robots," The International Journal of Robotics Research, vol. 28, no. 2, pp. 245-256, feb 2009.

[13] J. Maciejowski, Predictive Control with Constraints. Prentice-Hall, 2002.

[14] D. Dimitrov, A. Sherikov, and P.-B. Wieber, "Efficient resolution of potentially conflicting linear constraints in robotics," Aug. 2015, submitted to IEEE TRO (05/August/2015).

[15] A. Escande, N. Mansard, and P.-B. Wieber, "Hierarchical quadratic programming: Fast online humanoid-robot motion generation," The International Journal of Robotics Research, 2014.

[16] O. Kanoun, F. Lamiraux, and P.-B. Wieber, "Kinematic control of redundant manipulators: Generalizing the task-priority framework to inequality task," Robotics, IEEE Transactions on, vol. 27, no. 4, pp. 785-792, Aug 2011.

[17] P.-B. Wieber, R. Tedrake, and S. Kuindersma, Springer Handbook of Robotics. London: Springer London, 2015, ch. Modeling and Control of Legged Robots.

[18] C. Brasseur, A. Sherikov, C. Collette, D. Dimitrov, and P.-B. Wieber, "A robust linear MPC approach to online generation of $3 \mathrm{~d}$ biped walking motion," in 2015 IEEE-RAS 15th International Conference on
Humanoid Robots (Humanoids). Institute of Electrical \& Electronics Engineers (IEEE), nov 2015.

[19] P.-B. Wieber, "On the stability of walking systems," in Proceedings of the International Workshop on Humanoid and Human Friendly Robotics, Tsukuba, Japon, 2002.

[20] A. Herdt, N. Perrin, and P.-B. Wieber, "Lmpc based online generation of more efficient walking motions," in Humanoid Robots (Humanoids), 2012 12th IEEE-RAS International Conference on, Nov 2012, pp. 390395.

[21] A. Herdt, H. Diedam, P.-B. Wieber, D. Dimitrov, K. Mombaur, and M. Diehl, "Online Walking Motion Generation with Automatic Foot Step Placement,” pp. 719-737, 2010.

[22] P.-B. Wieber, "Trajectory free linear model predictive control for stable walking in the presence of strong perturbations," in IEEERAS International Conference on Humanoid Robots, Humanoids'06, December, 2006. Genova, Italie: IEEE, Dec. 2006, pp. 137-142.

[23] S. Al Homsi, A. Sherikov, D. Dimitrov, and P.-B. Wieber, "A hierarchical approach to minimum-time control of industrial robots," in IEEE International Conference on Robotics and Automation (ICRA), 2016, pp. 2368-2374.

[24] K. Kaneko, F. Kanehiro, S. Kajita, H. Hirukawa, T. Kawasaki, M. Hirata, K. Akachi, and T. Isozumi, "Humanoid robot hrp-2," in Robotics and Automation, 2004. Proceedings. ICRA '04. 2004 IEEE International Conference on, vol. 2, April 2004, pp. 1083-1090 Vol.2.

[25] H. J. Ralston, "Energy-speed relation and optimal speed during level walking," Internationale Zeitschrift für angewandte Physiologie einschließlich Arbeitsphysiologie, vol. 17, no. 4, pp. 277-283, 1958. 